\title{
STUDY OF THE EFFECT OF LEFT VENTRICULAR DIASTOLIC DYSFUNCTION ON PULMONARY HYPERTENSION IN CHRONIC SYSTOLIC HEART FAILURE AND ITS EFFECT ON PROGNOSIS
}

\author{
Subrata Basu1, Biswajit Haldar², Rakesh Sarkar³, Jayitri Majumdar, Sandip Ghosh ${ }^{5}$, Soma Biswas ${ }^{6}$, Manish Saha $^{7}$, Sukanya Bag ${ }^{8}$
}

${ }_{1}^{1}$ Assistant Professor, Department of Cardiology, North Bengal Medial College, Darjeeling, West Bengal, India.

${ }^{2}$ Associate Professor, Department of Pathology, North Bengal Medial College, Darjeeling, West Bengal, India.

${ }^{3}$ Fellow, Care Hospital, Hyderabad, Andhra Pradesh, India.

${ }_{4}^{4}$ Resident, Department of Paediatric Cardiology, NSH, Howrah, West Bengal, India.

${ }_{5}^{5}$ Assistant Professor, Department of Medicine, $R$ G Kar Medical College, Kolkata, West Bengal, India.

${ }^{6}$ Medical Officer, Department of Ophthalmology, B. N. Bose SD Hospital, Barakpur, Kolkata, West Bengal, India.

${ }^{7}$ Post Doctoral Resident, Department of Cardiology, R G Kar Medical College, Kolkata, West Bengal, India.

sPostgraduate Trainee, Department of Pathology, North Bengal Medial College, Darjeeling, West Bengal, India.

ABSTRACT
BACKGROUND
Pulmonary hypertension (PH) complicating left ventricular systolic dysfunction carries worse prognosis than LVSD alone. Though
nonlinear relation between the severity of the LVSD and the presence of PH exists, diastolic dysfunction (DD) may play some major
driving role in determining the degree of PH and henceforth may predict incremental prognostic information. This is a comparative
analysis of the parameters of different DD groups in determining the prognostication with special context to PH.

\section{MATERIALS AND METHODS}

Prospective analysis was conducted in patients with LVSD (EF-45\%) in the Department of Cardiology, R G Kar Medical College, Kolkata, for a period of two years. Clinical characteristics, biochemical parameters and quantitative Doppler measurement of pulmonary systolic pressure (PSP), indices of diastolic function were recorded and followed up for at least one year.

\section{RESULTS}

Out of a total 256, only 94 patients met the pre-specified criteria and different parameters were analysed. The patients are classified as mild, moderate and severe DD groups. There was significant difference in mean \pm SD of Heart rate, BMI and BNP level. Among the Doppler parameters only E/e is significantly ( $p$ value 0.01) different in these groups along with LVEF and PASP. 26 out of a total of 94 patients died in follow up period with Kaplan Meier survival curve for all cause death showing significant log rank test $\left(\chi^{2}\right.$ 15.94), with $p$ value $<0.001$ between these 3 groups. In multivariate regression analysis, age, PASP and TAPSE (Tricuspid Annular Peak Systolic Excursion) remain significant predictors of mortality whereas the ROC (Receiver Operating Curve) shows the E/e ratio as additional risk factor (AUC is 0.768 ).

\section{CONCLUSIONS}

Assessment of diastolic function provides added prognostic information in HFrEF. Though presence of PH increased the risk for clinical endpoints, the magnitude of diastolic dysfunction contributes to development of $\mathrm{PH}$ and has an impact on prognosis and overall mortality.

HOW TO CITE THIS ARTICLE: Basu S, Haldar B, Sarkar R, et al. Study of the effect of left ventricular diastolic dysfunction on pulmonary hypertension in chronic systolic heart failure and its effect on prognosis. J. Evolution Med. Dent. Sci. 2019;8(13): 1059-1063, DOI: $10.14260 /$ jemds/2019/234

\section{BACKGROUND}

Left ventricular systolic dysfunction (LVSD) plays an important role in the evolution of symptomatic congestive heart failure. The development of pulmonary venous or mixed pulmonary venous and arterial hypertension is an important pathophysiologic process in the progression from otherwise less severe left ventricular (LV) dysfunction to overt congestive heart failure. The frequency of $\mathrm{PH}$ is variable, In some clinical series, development of PH is

'Financial or Other Competing Interest': None.

Submission 26-01-2019, Peer Review 12-03-2019,

Acceptance 20-03-2019, Published 01-04-2019.

Corresponding Author:

Dr. Biswajit Haldar,

A-10/15,

Uttorayon, Matigara, Siliguri,

Darjeeling-734010,

West Bengal, India.

E-mail: biswajitpath@gmail.com

DOI: $10.14260 /$ jemds $/ 2019 / 234$

associated with an increased mortality/morbidity rather than isolated LV dysfunction without PH.1,2 The view of the contribution of left ventricular diastolic dysfunction (LVDD) as a determinant of pulmonary hypertension is controversial and not well defined as a tool in clinical practice. The role of DD on the evolution and course of congestive heart failure with preserved ejection fraction (HFpEF) was documented in several study, 3,4,5 whereas in the patients with reduced ejection fraction (HFrEF) is not well established. A study from Japan on 126 patients reported that the risk of hospital readmission and death increased with progressively increased diastolic dysfunction with patients having LVSD. ${ }^{6}$ Owan et al found that the clinical course and outcome is more favourable in patients with HFpEF than the patients with HFrEF.7

Doppler echocardiography (D-E) is an important adjunct for the assessment of patients with LV systolic dysfunction, not only for its non-invasive procedure and assessment of quantification of severity of pulmonary pressures, but it also provides indirect assessment of the of diastolic function. In previous studies, the parameters of diastolic function were 
found to be important independent determinants of filling pressures in the perspective of LVSD. ${ }^{8}$ The value of Dopplerderived indirect parameters of $\mathrm{LV}$ diastolic function in evaluation of LV filling pressures has been put into question. ${ }^{9}$ Hence, in routine clinical practice, the importance of Doppler derived parameters of diastolic function in patients with LVSD, especially the E/e ratio, remains to be elucidated.

\section{Aim of The Study}

The purpose of the study is comparative analysis of the parameters of different DD groups in determining the prognostication with special context to $\mathrm{PH}$.

\section{MATERIALS AND METHODS}

\section{Study Design, Setting and Patient Selection}

Patients attended in RGKMC with dyspnoea or known cases of heart failure under treatment were evaluated for LV systolic dysfunction. The selected cases, echo-Doppler derived diastolic parameters [ [mitral valve(MV] transmitral flow velocity (E)/mitral annular diastolic velocity(e) ratio (E/e)\}, MV deceleration time (DT), Isovolumetric Relaxation time (IVRT)], Left ventricular ejection fraction (LVEF) etc as well as estimation of Pulmonary systolic pressure (PSP) were done and the patients were kept under follow up for prognostication (By clinical worsening, hospitalisation, death due to cardiac cause etc.).

\section{Study Type and Design}

Prospective observational study.

\section{Study Place}

R. G. Kar. Medical College.

\section{Time Period}

1st January, 2012 to December, 2014.

\section{Sample Size}

94 patients.

Sample size has been taken based on convenience of the study

\section{Inclusion Criteria}

1. Age above 18 years.

2. left ventricular ejection fraction(LVEF) $<45 \%$;

3. PASP can be measurable by doppler echo using velocity of tricuspid regurgitation jet.

4. other doppler indices including tissue doppler derived parameters like mitral valve (MV) E/e ratio, MV deceleration time(MVDT), IVRT etc can be measurable by doppler echo.

\section{Exclusion Criteria}

1. Organic mitral, tricuspid, or aortic valvular disease or

2. History of any valve replacement or repair;

3. Infiltrative, constrictive, or hypertrophic cardiomyopathy.

4. Myocardial infarction within 6 months period.

5. Chronic obstructive lung disease or sleep apnoea;

6. Congenital heart disease;

7. Presence of tachyarrhythmias including atrial fibrillation.

8. Primary pulmonary arterial hypertension.
9. Prior chest radiation therapy;

10. Cardiac or lung transplantation.

11. Any concurrent chronic systemic disease/ infection like collagen vascular diseases, chronic HIV infection etc.

12. Patients unwilling to participate in the study.

Patients were followed up for 12 months for prognostic evaluation.

\section{Doppler Echocardiography}

A clinical, comprehensive two dimensional and doppler echo evaluation were done in all patients, ${ }^{10} \mathrm{LV}$ size and function was measured as per the standard recommendations of American Society, of Echocardiography. Continuous wave Doppler was used to assess maximal tricuspid regurgitation flow velocity to estimate the systolic pressure gradient between the right ventricle and right atrium. Right ventricular systolic pressure will then be calculated by adding an estimated right atrial pressure to the pressure gradient between RA\&RV.11 In the absence of any organic abnormality of the pulmonary or tricuspid valves this derived pressure will be considered to be identical to PASP. Early transmitral flow velocity (E) and early diastolic mitral annular velocity (e) will be measured with D-E in the apical 4chamber view with pulsed wave doppler and pulsed wave tissue doppler respectively to provide an estimate of LV diastolic function ${ }^{12}$. The ratio of peak (E) to peak (e) will be calculated (mitral E/e ratio) from the average of at least 3 cardiac cycles. The deceleration time of the E-wave and IVRT were also be measured. Left atrial volume index was calculated using the biplane area length method at end systole ${ }^{13}$. Severity of diastolic dysfunction if present is to be evaluated after considering different constellation parameters of diastolic function along with LA volume status.

\section{Statistical Analysis}

For continuous data, analysis was done as mean, SD or median with 25 th and 75th percentiles and for categorical data analysis was done as number and percentage. To compare continuous variables two-sample $t$ tests was used and signed-rank analysis was done to compare medians of values with nonparametric distributions, with statistical difference accepted for $\mathrm{p}<0.05$. Multiple linear regression analysis was utilised to evaluate associations among continuous clinical and two dimensional and Doppler echo derived variables with PSP. Analyses were done with the help of SPSS software 24 version.

\section{RESULTS}

\section{Clinical and Echo Characteristics}

Although larger number (256) of patients had initial requirement of LVEF of $<45 \%$, only 94 patients could be finally selected after fulfilling the study inclusion/exclusion criteria and having completed the stipulated echo evaluation and follow up. The patients of different grade of diastolic dysfunction (DD) have been compared using ANOVA and chi square test according to their clinico-demographical and echocardiographic parameter as shown in Table. 1. There is no statistical significance among these three groups in terms of age, sex and systemic BP, however it has been seen that elderly patients are mostly present in the severe DD group. They differ significantly in variation of heart rate and in BMI 
(p value 0.04 and 0.03 respectively) with incremental prognostic value with the grade of DD class. Difference of prevalence of diabetes and coronary artery disease (CAD) not significantly identified though the revascularisation is mostly present in moderate and severe DD group. Patients were receiving standard oral $\mathrm{HF}$ medication which were fortnightly/monthly supervised showed no difference of distribution of dose of medicine except the angiotensin receptor and neprilysin inhibitor (ARNI) which was mostly prescribed for moderate and severe groups(26\% Vs. 58\%, Vs $60.3 \%$ p0.02) and the Digoxin (31\% Vs 38\% Vs $46 \%$, p0.04).There was not remarkable difference in status of creatinine clearance i.e. GFR value (p0.17)but significant difference $(p<0.01)$ in terms of BNP level(measured before the initiation of any novel medication e.g. ARNI). Several Doppler derived hemodynamic parameters were also compared and most notable were mean EF ( $p$ 0.014), PASP ( $p$ value 0.04 ) and MV E/e (p 0.01).

\begin{tabular}{|c|c|c|c|c|}
\hline & $\begin{array}{c}\text { Mild } \\
\mathrm{N}=28\end{array}$ & \begin{tabular}{|c|} 
Moderate \\
$\mathrm{N}=35$
\end{tabular} & $\begin{array}{c}\begin{array}{c}\text { Severe } \\
\mathrm{N}=31\end{array} \\
\end{array}$ & $\begin{array}{c}\text { p } \\
\text { Value }\end{array}$ \\
\hline Age, Yrs. & $52.6 \pm 4.6$ & $58.3 \pm 6.2$ & $59.6 \pm 7.8$ & 0.263 \\
\hline Sex,\% Male & 61 & 70.3 & 55.1 & 0.175 \\
\hline BMI, Kg/m² & 24.5 & 21.7 & 20.8 & 0.034 \\
\hline $\begin{array}{c}\text { Systemic BP, } \\
\text { mmHg }\end{array}$ & $\begin{array}{c}\text { Systolic- } \\
124 \pm 21 \\
\text { Diastolic- } \\
71 \pm 12 \\
\end{array}$ & $\begin{array}{c}119 \pm 17 \\
74 \pm 6\end{array}$ & $\begin{array}{c}126 \pm 15 \\
72 \pm 11\end{array}$ & 0.102 \\
\hline $\begin{array}{l}\text { HR, Beats per } \\
\text { Min }\end{array}$ & $78 \pm 12$ & $83 \pm 14$ & $87 \pm 11$ & 0.045 \\
\hline \begin{tabular}{|c|}
$\mathrm{H} / \mathrm{O}$ CAD/ \\
Revascularisation
\end{tabular} & $68.6 \%$ & $73.1 \%$ & $72.4 \%$ & 0.07 \\
\hline $\begin{array}{c}\text { Diabetes Mellitus, } \\
\mathrm{n}(\%)\end{array}$ & $17(60.7 \%)$ & $20(70 \%)$ & $21(67.7 \%)$ & 0.08 \\
\hline $\mathrm{GFR}, \mathrm{ml} / \mathrm{min}$ & $75.8 \pm 45$ & $81.5 \pm 37$ & $79.5 \pm 61$ & 0.17 \\
\hline BNP, pg/ml & $302 \pm 201$ & $462 \pm 183$ & $704 \pm 374$ & 0.01 \\
\hline LVEF, $\%$ & $35.3 \pm 17$ & $30.6 \pm 12.4$ & $28.3 \pm 11.2$ & 0.014 \\
\hline LAVI & $40.5 \pm 16.6$ & $42.4 \pm 21.3$ & $43.7 \pm 27.2$ & 0.08 \\
\hline MV E/e & $10.6 \pm 8.2$ & $18.2 \pm 11.4$ & $21.7 \pm 6.9$ & 0.01 \\
\hline PASP, $\mathrm{mmHg}$ & $42.6 \pm 31.2$ & $49.4 \pm 26.8$ & 56.32 .9 & 0.04 \\
\hline \multicolumn{5}{|c|}{$\begin{array}{c}\text { Table 1. Comparative Analysis of Clinical, Demographic } \\
\text { and Echocardiographic Profile of Patients with Mild, } \\
\text { Moderate and Severe LV Diastolic Dysfunction in Patients } \\
\text { with EF }<45 \%\end{array}$} \\
\hline
\end{tabular}

\section{Midterm Outcome}

Out of 94 patients 48 patients remained stable after clinical and hemodynamic parameter (Evaluated by Doppler) study whereas 32 got worsened and 14 patients actually showed improvement in follow up period of at least 1 year. Out of these 32 patients 26 succumbed. The cumulative survival curve in different DD classes as shown in the Fig.1 demonstrate that the mortality is significantly varying among these three groups with Breslow log rank (Generalised Wilcoxon) test $\chi^{2} 15.94$ ( $\mathrm{p}$ value $<0.001$ ).
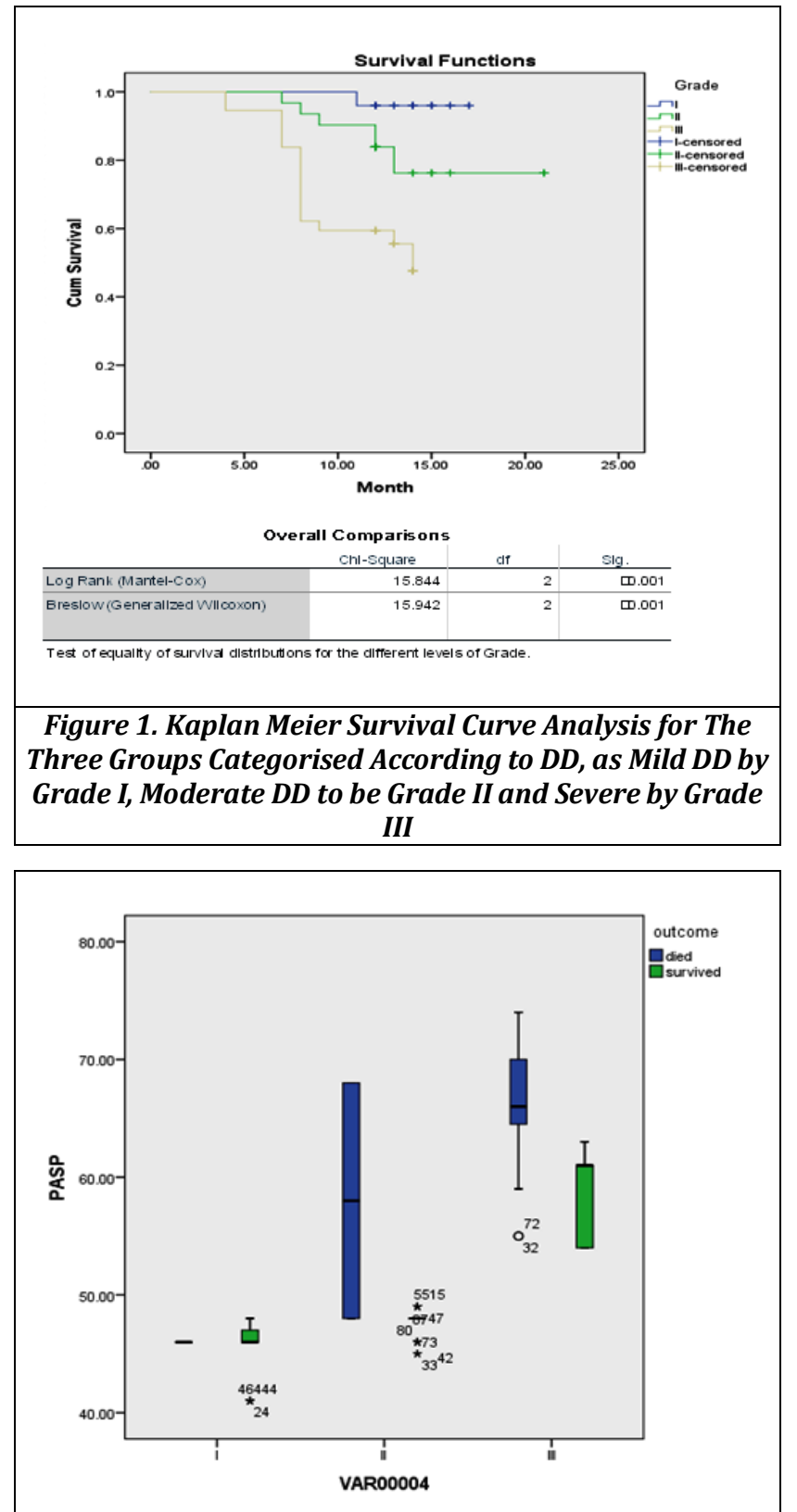

Figure 2. Boxplot Distribution of Median Value of PASP in Different DD Groups in Relation to Survival Outcome

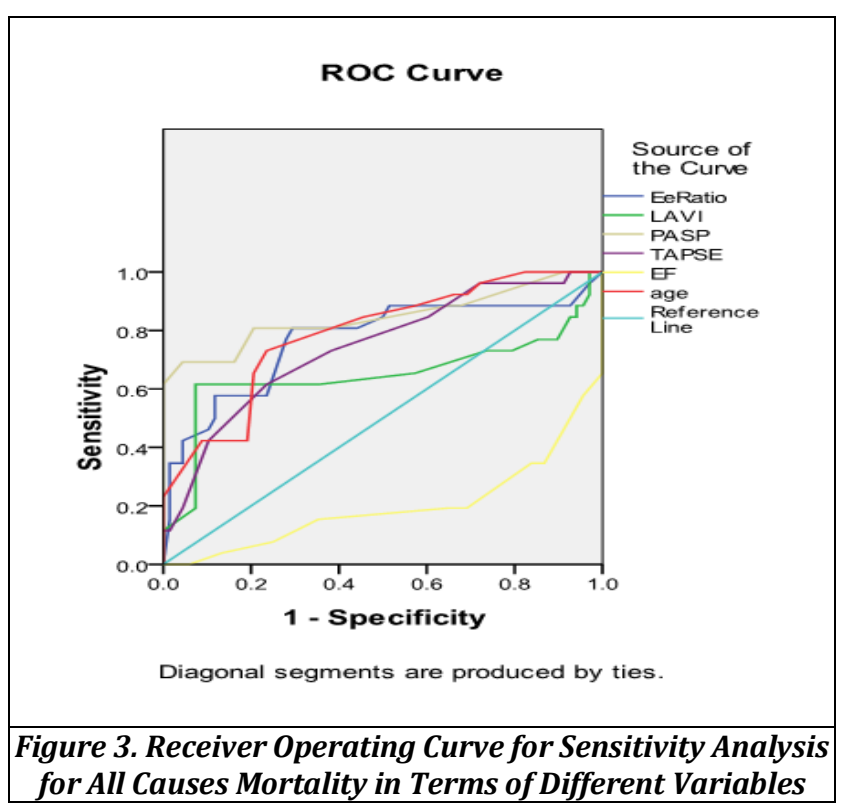


Prediction of Outcome

\begin{tabular}{|c|c|c|c|c|c|c|}
\hline & & B & S.E. & Wald & df & Sig. \\
\hline \multirow{4}{*}{ Step 1a } & EF & -0.521 & 0.371 & 1.970 & 1 & 0.160 \\
\cline { 2 - 7 } & PASP & -0.509 & 0.204 & 6.201 & 1 & 0.013 \\
\cline { 2 - 7 } & Age & -0.422 & 0.149 & 7.998 & 1 & 0.005 \\
\cline { 2 - 7 } & Gender(1) & 1.918 & 1.668 & 1.322 & 1 & 0.250 \\
\cline { 2 - 7 } & TAPSE & 0.362 & 0.171 & 4.482 & 1 & 0.034 \\
\cline { 2 - 7 } & Constant & 67.281 & 28.486 & 5.579 & 1 & 0.018 \\
\hline
\end{tabular}

Table 2. Multivariate Regression Model Analysis for Risk Prediction for Mortality

\section{Gender (1) for Male}

In multivariate regression model age, PASP and TAPSE have been identified as significant(p value $0.005,0.013$ and 0.034 ) predictor of mortality as demonstrated in table 2 . The distribution of median value of PASP is shown in Fig. 2 in different DD groups in relation to survival outcome.

\section{Sensitivity Analysis of Predictors}

The sensitivity analysis of the covariates in ROC system Fig. 3 shows that PASP of cut off level of $51.5 \mathrm{mmHg}$ has sensitivity of $80 \%$ and specificity of near $70 \%$ and cut off for TAPSE of 16.5 has sensitivity of $>70 \%$ and specificity of $62 \%$ using c statistics. ROC analysis demonstrates the E/e and LAVI as additional risk factor with AUC is 0.768 and 0.648 (p value of $<$ 0.001 and 0.027 .

\section{DISCUSSION}

Our study shows that the patients with SD had an incremental impact of diastolic dysfunction on clinical outcome. Whereas majority studies showed that the severity of left ventricular systolic dysfunction was the key prognostic marker, but diastolic dysfunction had an important role in modifying the clinical outcome, hereby opened an important dimension rendering in research works consequent necessary therapeutic intervention. Luers et al. in their study showed that DD had incremental effect on prognosis in patients with less severe SD compared to patients with more severe SD. ${ }^{14}$ Kuijk et al, in their study showed that in the patients with peripheral arterial disease, the increased cardiovascular mortality and morbidity was associated independently with presence of isolated diastolic, systolic, or combined LV dysfunction irrespective of the presence of other risk factor (e.g. diabetes mellitus etc.). Patients with combined systolic and diastolic dysfunction had the worst cardiovascular prognosis. ${ }^{15}$ Lee et al showed that a decrease of an initially high e/a ratio predicted a more favorable long term clinical outcome with therapy in otherwise clinically stable patients with chronic HF and severe LVSD ${ }^{16}$ Meluzin et al, studied a systolic and a diastolic parameter of RV function, the peak systolic tricuspid annular velocity, and peak early diastolic tricuspid annular velocity respectively for delineating prognostic value of patients with heart failure. The authors categorized patients into 4 groups with different combination of the parameters of diastolic function and with different risk of cardiac events and death. It is already known that the impaired relaxation of ventricle due to increased stiffness which is the prime mechanism of DD often leads to the compromised state of cardiac performance status particularly the cardiac output during exercise or on exertion.
This failing response of contractile function may be one of the precursor of systolic dysfunction which can be shown by the deterioration of the systolic longitudinal strain. ${ }^{17,18}$ This might explain the issue of incremental relative risk of additional DD irrespective of the degree of SD. ${ }^{19}$ On the other hand, in severe LVSD, pathophysiological processes postulated, may well coincide with those responsible for diastolic dysfunction. Whether a severely compromised LVEF always represents the final common pathway of exaggerated systolic and diastolic dysfunction, is yet to be established.20,21

An increasing number of studies have assessed the risk of readmission and mortality in patients with HFrEF-related $\mathrm{PH}$ over the last decade, and mostly in North America and Europe. In a systemic review of literature on predictors of mortality and hospitalization of patients of $\mathrm{PH}$ with left heart disease in 24 studies out of 26, PH has been shown to be an independent risk factor, however uniformity is lacking in association between magnitude of reduction of EF and the presence of $\mathrm{PH}$ and the mortality. ${ }^{22}$ In addition to effect of $\mathrm{PH}$; age, NYHA class, decreased GFR and RV function were also integrated in the multivariable analysis for outcome risk in this review. ${ }^{23}$

\section{Study Limitation}

In presence of systolic dysfunction, the present study did not investigate the validity of the echocardiographic diastolic measurements. On the other hand, there is no head to head comparison with the reference standard was available (i.e., invasive hemodynamic assessment). The study population is very small as well as the duration of follow up period for reflecting statistical significance to draw any conclusion.

\section{CONCLUSION}

The present study shows that the degree of diastolic dysfunction in patients with HFrEF modifies the status of $\mathrm{PH}$ by change of LV filling pressure and provides further prognostic information particularly in presence of RV dysfunction irrespective of degree of LVEF.

\section{REFERENCES}

[1] Kjaergaard J, Akkan D, Iversen KK, et al. Prognostic importance of pulmonary hypertension in patients with heart failure. Am J Cardiol 2007;99(8):1146-50.

[2] Ghio S, Gavazzi A, Campana C, et al. Independent and additive prognostic value of right ventricular systolic function and pulmonary artery pressure in patients with chronic heart failure. J Am Coll Cardiol 2001;37(1):183-8.

[3] Redfield MM, Rodeheffer RJ, Jacobsen SJ, et al. Plasma brain natriuretic peptide to detect preclinical ventricular systolic or diastolic dysfunction: a community based study. Circulation 2004;109(25):3176-81.

[4] Lam CS, Lyass A, Kraigher-Krainer E, et al. Cardiac dysfunction and non-cardiac dysfunction as precursors of heart failure with reduced and preserved ejection fraction in the community. Circulation 2011;124(1):24-30.

[5] Luers C, Wachter R, Kleta S, et al. Natriuretic peptides in the detection of preclinical diastolic or systolic dysfunction. Clin Res Cardiol 2010;99(4):217-26. 
[6] Hirata K, Hyodo E, Hozumi T, et al. Usefulness of a combination of systolic function by left ventricular ejection fraction and diastolic function by E/E0 to predict prognosis in patients with heart failure. Am J Cardiol 2009;103(9):1275-9.

[7] Owan TE, Hodge DO, Herges RM, et al. Trends in prevalence and outcome of heart failure with preserved ejection fraction. $\mathrm{N}$ Engl J Med 2006;355(3):251-9.

[8] Enriquez-Sarano M, Rossi A, Seward JB, et al. Determinants of pulmonary hypertension in left ventricular dysfunction. J Am Coll Cardiol 1997;29(1):153-9.

[9] Mullens W, Borowski AG, Curtin RJ, et al. Tissue Doppler imaging in the estimation of intracardiac filling pressure in decompensated patients with advanced systolic heart failure. Circulation 2009;119(1):62-70.

[10] Tsang TS, Barnes ME, Gersh BJ, et al. Prediction of risk for first age-related cardiovascular events in an elderly population: the incremental value of echocardiography. Journal of the American College of Cardiology 2003;42(7):1199-205.

[11] Hurrell DG, Nishimura RA, Ilstrup DM, et al. Utility of preload alteration in assessment of left ventricular filling pressure by Doppler echocardiography: a simultaneous catheterization and Doppler echocardiographic study. J Am Coll Cardiol 1997;30(2):459-67.

[12] Khankirawatana B, Khankirawatana S, Peterson B, et al. Peak atrial systolic mitral annular velocity by Doppler tissue reliably predicts left atrial systolic function. Journal of the American Society of Echocardiography 2004;17(4):353-60.

[13] Nagueh SF, Sun H, Kopelen HA, et al. Hemodynamic determinants of the mitral annulus diastolic velocities by tissue Doppler. J Am Coll Cardiol 2001;37(1):27885.

[14] Lüers C, Edelmann F, Wachter R, et al. Prognostic impact of diastolic dysfunction in systolic heart failure-a cross-project analysis from the German Competence Network Heart Failure. Clinical Cardiology 2017;40(9):667-73.
[15] Van Kuijk JP, Flu WJ, Valentijn TM, et al. Influence of left ventricular dysfunction (diastolic versus systolic) on long-term prognosis in patients with versus without diabetes mellitus having elective peripheral arterial surgery. Am J Cardiol 2010;106(6):860-4.

[16] Lee $\mathrm{CH}$, Hung $\mathrm{KC}$, Chang $\mathrm{SH}$, et al. Reversible left ventricular diastolic dysfunction on Doppler tissue imaging predicts a more favorable prognosis in chronic heart failure. Circ J 2012;76(5):1145-50.

[17] Phan TT, Abozguia K, Shivu GN, et al. Myocardial contractile inefficiency and dyssynchrony in heart failure with preserved ejection fraction and narrow QRS complex. J Am Soc Echocardiogr 2010;23(2):2016.

[18] Kraigher-Krainer E, Shah AM, Gupta DK, et al. Impaired systolic function by strain imaging in heart failure with preserved ejection fraction. J Am Coll Cardiol 2014;63(5):447-56.

[19] Motoki H, Borowski AG, Shrestha K, et al. Incremental prognostic value of assessing left ventricular myocardial mechanics in patients with chronic systolic heart failure. J Am Coll Cardiol 2012;60(20):2074-81.

[20] Phan TT, Abozguia K, Shivu NG, et al. Heart failure with preserved ejection fraction is characterized by dynamic impairment of active relaxation and contraction of the left ventricle on exercise and associated with myocardial energy deficiency. J Am Coll Cardiol 2009;54(5):402-9.

[21] Van Heerebeek L, Borbely A, Niessen HW, et al. Myocardial structure and function differ in systolic and diastolic heart failure. Circulation 2006;113(16):1966-73.

[22] Dzudie A, Kengne AP, Thienemann F, et al. Predictors of hospitalizations for heart failure and mortality in patients with pulmonary hypertension associated with left heart disease: a systematic review. BMJ Open 2014;4(7):e004843.

[23] Wang D, Han Y, Zang H, et al. Prognostic effects of pulmonary hypertension in patients undergoing cardiac resynchronization therapy. J Thorac Dis 2010;2(2):71-5. 